\title{
Cocaine induced gastric ischemia
}

\section{Abstract}

A 50year-old male presented to the emergency department with a 2-3month history of intermittent hematemesis accompanied by epigastric constant pressure, worsened by oral intake. He also complained of bowel movements admixed with bright and dark blood. There was an associated 30pound weight loss over the last 5 months. He denied any fevers, night sweats, shortness of breath, or dizziness. He was not on any anti-platelet or anticoagulation treatment. An esophagogastroduodenoscopy performed in 2006 revealed gastric and esophageal ulcers. He did admit to a recent history of tobacco, alcohol, and cocaine use. His vital signs were within normal limits. On physical exam, his abdomen was distended with mild epigastric tenderness and no peritoneal signs. Placement of a nasogastric tube resulted in the drainage of bloodtinged fluid. Laboratory results on admission revealed a white count of $7.7 \mathrm{k} / \mathrm{uL}$ with mild absolute eosinophilia, hemoglobin of $16.8 \mathrm{~g} / \mathrm{dL}$, hypokalemic hypochloremic metabolic alkalosis (potassium $3.4 \mathrm{mmol} / \mathrm{L}$, chloride $95 \mathrm{mmol} / \mathrm{L}$, bicarbonate $38 \mathrm{mmol} / \mathrm{L}$ ). Liver function tests, lipase and anticoagulation profile were normal. Serum alcohol level was elevated at $168 \mathrm{mg} / \mathrm{dL}$. Urine toxicology screen on admission was positive for cocaine. A computed tomographic scan of his abdomen/pelvis with intravenous contrast is shown in Figure 1. The patient was taken to the operating room shortly after the scan.

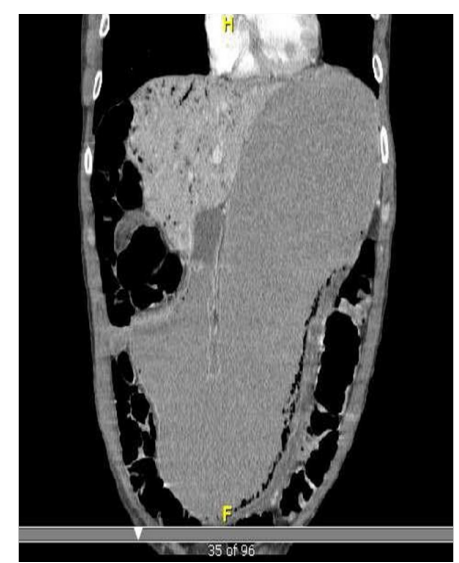

Figure I A computed tomographic scan of his abdomen/pelvis with intravenous contrast.

Keywords: cocaine, ischemic gastritis, pneumatosis, portal venous gas
Volume 5 Issue I - 2018

\author{
Alisha Gupta,' Julietta Chang,, Mihir M Shah ${ }^{3}$ \\ 'Department of Internal Medicine,Allegheny General Hospital, \\ USA \\ ${ }^{2}$ Department of General Surgery, Cleveland Clinic Foundation, \\ USA \\ ${ }^{3}$ Division of Surgical Oncology, Rutgers Cancer Institute of New \\ Jersey, USA
}

Correspondence: Mihir M Shah, Division of Surgical Oncology, Rutgers Cancer Institute of New Jersey, 195 Little Albany St, USA,Tel (732) 2356780, Email shahmihir_999@hotmail.com

Received: November 30, 2017| Published: January 24, 2018

\section{What is the diagnosis?}

\section{i. Ischemic Gastritis \\ ii. Necrosis of the Small Intestine \\ iii. Perforated Gastric Ulcer \\ iv. Gastric Volvulus}

Pneumatosis intestinalis (PI) describes the presence of gas-filled cysts within the wall submucosa and subserosa of the bowel. ${ }^{1}$ Many theories on the etiology of this condition exist, and potential sources of gas include-1. intraluminal gas entering the mucosa secondary to increased intraluminal pressure; 2. bacterial production of $\mathrm{H} 2$ gas, where the tension of $\mathrm{H} 2$ is greater than the tension of nitrogen in the blood, causing diffusion of gas from the gut lumen towards submucosal vessels; 3. pulmonary gas, where gas from ruptured alveoli tract along vasculature in the mediastinum caudally to the retroperitoneum and bowel mesentery. ${ }^{2}$ The gas can embolize from the bowel wall through the mesenteric veins to the portal venous system and the non-dependent parts of the liver. Thus, benign etiologies exist for pneumatosis and studies suggest that half of all patients with PI on CT were successfully managed non-operatively. ${ }^{3-5}$ Findings of abdominal distension on physical exam and dilated loops of bowel on $\mathrm{CT}$, lactic academia, and peritonitis are uncommon in patients managed non-operatively. ${ }^{5}$ Although the presence of portal venous gas should heighten concern, it should not always mandate surgery, ${ }^{5}$ but be viewed in the context of the entire condition of the patient.

Our patient's CT was read as PI along the greater curvature and antrum with $15 \mathrm{~mm}$ thickened gastric wall and air within the portal venous system. He had gross evidence of hemorrhagic gastritis without necrosis or perforation during the operation (diagnostic laparoscopy and upper endoscopy with gastric biopsy). Patient was kept NPO, on IV antibiotics, TPN with nasogastric decompression. Our plan was to rescan him in a week if he continues to progress well. However, he left against medical advice on the fifth post-operative day. Pathology 
from the upper endoscopy with biopsy confirmed the findings of ischemic gastritis. He returned for a follow-up sevenmonths later in the outpatient clinic, and a repeat CT scan demonstrated gastric distension, without gastric wall thickening or pneumatosis. He has been lost to follow-up after this repeat CT. His likely cause of ischemic gastritis was cocaine abuse.

\section{Acknowledgements}

None.

\section{Conflict of interest}

Author declares that there is no conflict of interest.

\section{References}

1. Adam. Grainger \& Allison's Diagnostic Radiology. 5th ed. USA; 2014. p. 599-601.

2. Khalil PN, Huber-Wagner S, Ladurner R, et al. Natural history, clinical pattern, and surgical considerations of pneumatosis intestinalis. Eur $J$ Med Res. 2009;14(6):231-239.

3. Morris MS, Gee AC, Cho SD, et al. Management and outcome of pneumatosis intestinalis. Am J Surg. 2008;195(5):679-683.

4. Hawn MT, Canon CL, Lockhart ME, et al. Serum lactic acid determines the outcomes of CT diagnosis of pneumatosis of the gastrointestinal tract. Am Surg. 2004;70(1):19-24.

5. Duron VP, Rutigliano S, Machan JT, et al. Computed Tomographic Diagnosis of Pneumatosis Intestinalis. Arch Surg. 2011;146(5):506-510. 